\title{
Ethical issues concerning cardiac surgery in elderly patients - the nurse's role as a patient advocate: A case report
}

\section{Corresponding author:}

Izabella Uchmanowicz Wroclaw Medical University, Faculty of Health Sciences, Department of Clinical Nursing, Bartla 5 Str. 51-618 Wroclaw, Poland. e-mail: izabella.uchmanowicz@umed.wroc.pl

Medical Research Journal 2020; Volume 5, Number 2, 120-125 10.5603/MRJ.a2020.0011 Copyright (C) 2020 Via Medica ISSN 2451-2591

\begin{abstract}
Introduction: Frailty syndrome (FS) is one of the well-known risk factors for cardiac surgical treatment. Moreover, older patients are more likely to suffer from various comorbidities. Ethical issues that arise in patient care should be considered, including their identification, analysis, and appropriate resolution. The study aimed to present the nurse's role in the therapeutic team, which should take the floor as a patient advocate representing her/his interest.

Case presentation: An 82-year-old patient was admitted to the Cardiac Surgery Clinic and was discussed in the context of emerging ethical dilemmas in clinical practice. The peri-operative risk was assessed as high; the nurse identified both frailty phenotype and FS. Currently, at postoperative day 40, the patient remains sedated, haemodynamically unstable, and has a poor long-term prognosis.

Summary: If the patient is not presented with a risk assessment that includes FS assessment during the qualification process, it can be concluded that this omission violates the information component of informed consent. Nurses must speak out in those patients' interests in order to preclude actions that may increase their vulnerability during cardiac surgery.

Key words: cardiac surgery, frailty syndrome, patient advocacy, case report
\end{abstract}

Med Res J 2020; 5 (2): 120-125

\section{Introduction}

The profile of patients who are qualified for cardiac surgery has changed due to two factors. First is the systematic increase in life expectancy; it is estimated that in 2050, people over 65 years old may constitute $21 \%$ of the entire population [1]. Second is medical developments, including improvements in medical equipment, diagnostics, and surgical and therapeutic techniques. Due to these factors, over the past 15 years, the average age of patients treated with cardiac surgery has increased from 56 years to 69 years, and $10 \%$ of them are aged 80 years or older [2]. Because patient vulnerability increases with age, geriatricians divide elderly people into three age groups: young old (65 75 years), old old (75 85 years), and oldest old (over 85 years) [3]. Nevertheless, advanced age no longer automatically confers the assumption that a patient is at increased risk of adverse medical events [4].
Many studies have proven that elderly patients can benefit from cardiac surgery [2]. However, the fact is that the older the patient, the more likely the presence of comorbidities [1]. Each older person has an average of four chronic diseases, and each year, nearly $20 \%$ of people in this age group are hospitalised [5]. In cardiac surgery, the increased risk of mortality, morbidity, or use of additional resources is not associated with age itself but with the coexistence of chronic diseases that carry numerous risk factors. In addition, it is now recognised that giving older people lower priority for operative medical care violates the principle of respect for human dignity unless their age decreases the chance of benefitting from treatment and care [6]. Therefore, any medical decisions that take into account non-medical criteria, including the patient's social status, material resources, or age, are considered ethically wrong [7].

The current procedure of qualifying patients for cardiac surgery assesses perioperative risk based on 
two calculators widely used in clinical practice: The Society of Thoracic Surgeons (STS) risk score and the EuroSCORE II (ES II). In addition to age and gender, both calculators include in their assessment a number of clinical factors that may increase patient mortality after cardiac surgery. However, these calculators do not assess psychosocial factors, which in light of current knowledge is a limitation. The ES II typically overestimates peri-operative risk, whereas the STS typically underestimates it; therefore, the use of the latter calculator should be considered in patients who have frailty syndrome (FS) [8]. Clinicians believe that risk indicators are helpful but are only one factor in making decisions and setting medical priorities [6]. When decisions are made, the relationship between treatment benefit and possible complications should always be positive [1].

Old age is no longer the main exclusion criterion used in qualifying patients for cardiac surgery, although scientific research considers FS to be a factor that negatively affects cardiac surgery. Because clinical practice still lacks a standardised model for qualifying FS patients for cardiac surgery, care planning must be scrupulous for both medical and ethical reasons. The emergence of ethical dilemmas is an integral part of clinical practice. Clinical ethics addresses identifying, analysing, and solving moral problems that arise in patient care [5].

Ethical dilemmas arise even in optimal settings, and in cardiac surgery they are important from both the clinical and scientific points of view [9]. Given the ongoing medical developments and emergence of new concepts, clinical ethics must be a dynamic and constantly evolving discipline [10]. Likewise, every medical intervention must scrupulously observe basic ethical principles, including autonomy, beneficence, non-maleficence, and justice [5]. Nearly $80 \%$ of clinicians declare that they have abstained from surgical treatment in their professional practice because the risk was too high in relation to the benefits, and $26 \%$ have declared that they were guided in their decision to abstain by ethical principles [6].

The aim of this case study was to present a clinical situation in which an elderly patient with high perioperative risk and identified FS was qualified for elective surgery. How should a nurse behave when he or she has information that the presence of FS may make the risk of surgery higher than it has been represented to the patient? The changing profile of patients who are qualified for cardiac surgery may generate an ethical dilemma and creates the need for strategies that are appropriate and consistent with the principles of ethics. The main purpose of this paper is to illustrate the importance of the nurse's role in the therapeutic team, which may include speaking out in a patient's best interests as an advocate. A nurse should always speak out within the scope of his or her competence, but as a member of a multidisciplinary team, a nurse can significantly influence the clinical decision making. A nurse must be ethically sensitive and recognise cases in which planned medical interventions will not necessarily benefit the patient. In addition, this paper discusses issues related to $\mathrm{FS}$, assessing peri-operative risk, the presence of depressive symptoms, the essence of social support, decision-making capability, the decision-making process, the essence of communication, and the emerging practice of prehabilitation.

\section{Case report}

This case report study was approved by the independent Bioethics Committee of Wroclaw Medical University, Poland blinded source. After receiving oral and written explanations of the study, the patient gave written, informed consent prior to starting the study. The case study was carried out in compliance with the protocol of Good Clinical Practices and Declaration of Helsinki principles. This case report adheres to CAse REporting (CARE) guidelines [11].

An 82-year-old patient was admitted voluntarily to the Cardiac Surgery Clinic in Wroclaw, Poland blinded source for the purpose of undergoing mitral valve replacement surgery, aortic valve reconstructive surgery, and coronary-artery bypass grafting. The comorbidities were permanent atrial fibrillation, ischaemic heart disease, previous pacemaker implantation, arterial hypertension, anaemia (treated), hyperthyroidism, and chronic gastritis.

During the heart group meeting, the peri-operative risk was assessed at $7.12 \%$ using the ES II calculator. In addition, the purpose of the procedure, its scope, course, and the possibility of postoperative complications were discussed with the patient, who signed the consent form to conduct the procedure. At the time of her admission, the patient was in New York Heart Association (NYHA) class III, ejection fraction (EF) 50\%.

The day before the procedure, the nurse, after obtaining written consent to do so, included the patient in a study aimed at identifying FS. During an interview, the patient stated that she had recently become a widow after having cared for her disabled husband for 15 years. In addition, a few months earlier, the patient's only son had died suddenly of a heart attack. The patient expressed that she could not cope mentally with it.

The nurse used standardised tools to assess the patient's cognitive state and to determine the frailty phenotype, including assessing symptoms that can indicate depression. Based on the interpretation of the results, cognitive disorders without dementia were identified (24 points) on the Mini-Mental State Exam- 
Table 1. Results for the assessment of the patient's frailty phenotype according to Fried criteria

\begin{tabular}{lc}
\hline Frailty Phonotype - Criteria & Results \\
\hline Shrinking: unintentional weight loss $>4.54 \mathrm{~kg}$ per year & $7.0 \mathrm{~kg}$ per year \\
Weakness: grip strength in the lowest $20 \%$, stratified by gender and BMl $\left(\mathrm{kg} / \mathrm{m}^{2}\right)$ & $17.0 \mathrm{~kg}$ \\
Exhaustion: self-reported exhaustion, identified by two questions from the CES-D scale & $\mathrm{M}=2.2$ points \\
Slowness: based on time to walk $4.57 \mathrm{~m}$, adjusting for gender and standing height & $4.74 \mathrm{~s}$ \\
Low physical activity: a weighted score of kilocalories expended per week, stratified by gender & $200 \mathrm{kcal}$ \\
\hline
\end{tabular}

$\mathrm{M}$ - the mean scoring; BMI — body mass index; CES-D - Centre for Epidemiologic Studies - Depression. Positive for frailty phenotype $\geq 3$ criteria present; Intermediate or prefrail: 1 or 2 criteria present

Table 2. Results obtained in the biopsychosocial domains of the Tilburg Frailty Indicator

\begin{tabular}{lc}
\hline Tilburg Frailty Indicator (TFI) & Results \\
\hline Total score & 11 points \\
The physical domain (0-8 points) & 6 points \\
The psychological domain (0-4 points) & 3 points \\
The social domain (0-3 points) & 2 points \\
\hline
\end{tabular}

*The higher the score, the higher the patient's frailty. Frailty syndrome is diagnosed when the total TFI score is $\geq 5.13$ points

ination (MMSE) questionnaire. A frailty phenotype was identified according to Fried (Tab. 1) [12], and FS was identified according to the Tilburg Frailty Indicator (TFI) (Tab. 2) [13, 14].

The next day, the patient underwent the complex cardiac surgery and currently is at post-operative day 40. The patient remains sedated, haemodynamically unstable, and breathing with mechanical assistance. She is haemodialysed periodically and has a poor long-term prognosis.

\section{Discussion}

In medicine, the essence of any decision-making process is communication. Randomised trials have proven that effective communication between patient and clinician results in greater satisfaction and fulfilment and improved medical results. In addition, communication can prevent and resolve ethical dilemmas arising in clinical practice. Effective communication is an integral part of the medical arts and prevents or mitigates ethical dilemmas arising during the decision-making process. Healthcare providers are responsible for making patients and their families aware that any information conveyed before cardiac surgery can be important to the decision-making process. Mueller et al. [5] listed topics that are crucial to analysing ethical dilemmas related to specific cases. These include medical indications (beneficence, non-maleficence), patient preferences (respect for patient autonomy), quality of life (beneficence, non-maleficence, respect for patient autonomy), and contextual features (loyalty, justice).

This raises the following question: What is the role of the nurse in the decision-making process? As a permanent member of a multidisciplinary team, a nurse is obligated to speak up on issues related to his or her professional competence and is also a link in the communication channel that includes the doctor, other members of the multidisciplinary team, and the patient and/or the patient's family (Fig. 1). In recent years, patient advocacy has become an intensely investigated issue in the nursing field. In fact, patient advocacy has been identified as an essential activity of nursing [15]. The tasks of nurses include protecting patients from injuries and potential risks, whether mental or physical and whether intentional or unintentional, that are associated with improper treatment or care [16]. Given the numerous factors that may increase the vulnerability of patients during cardiac surgery, a nurse, despite the necessity of rationing his or her care, must constantly be aware of the biopsychosocial status of his or her patients. In the study case reported herein, the nurse used available standardised methods to assess FS, and the inclusion of the FS concept widened the perception of the patient's psychosocial dimension. Comprehensive pre-operative assessment is vital to determining not only individual peri-operative risk but also the potential benefits of treatment to the group of which that individual is a member. 


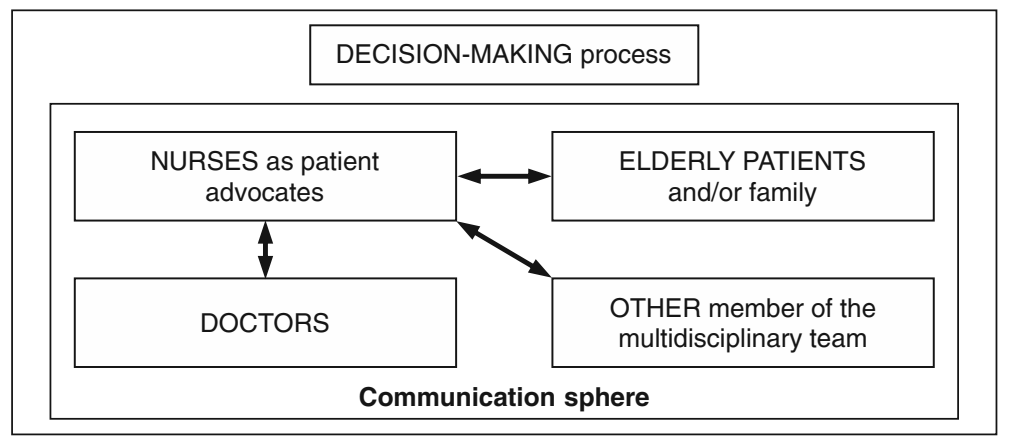

Figure 1. The place and role of the nurse in the decision-making process

In cardiac surgery, after comorbidities, FS is the strongest predictor of mortality and morbidity, stronger than patient age. Cardiac surgery patients are in a dynamic state, and frailty increases vulnerability to stressors that are often clinically associated with the occurrence of postoperative complications and the need for intensive medical care. The hospitalisation cost for a cardiac-surgery patient with FS is $30 \%$ higher than for a non-frail patient [17]. A review of the literature on the effects of FS on cardiac surgery identified a relationship between FS and adverse post-operative results: patients who had been identified with FS were more likely to develop mortality, morbidity, and functional impairment, i.e. major adverse cardiac and cerebrovascular events (MACCE) [8].

Despite an increasing number of studies on the negative effects of FS on cardio-surgical treatment, its systematic assessment still is not included in daily clinical practice. As one article in the literature stated, 'Patient frailty is the elephant in the operating room: it is easy to spot but is often ignored' [3]. Many standardised tools for identifying FS exist. However, there is still no unified model of risk forecasting that includes FS assessment, which is an important factor from the point of view of optimising the effectiveness of therapy. According to Afilalo [18], who assessed the prognostic value of various frailty-assessment tools, only five-metre gait speed is significantly associated with complications or a two- to three-fold increase in the risk of mortality. Hill et al. [19] recommended adding to the decision-making process a peri-operative risk-assessment model, the first stage of which identifies FS by assessing cognitive abilities, gait, nutritional status, and the basic and complex activities of daily life. In the study case described herein, FS was assessed using two standardised tools that are recommended by consensus [20]. Unfortunately, the peri-operative risk that was presented to the patient during the heart group meeting did not include the diagnosis of FS because the FS evaluation was only part of another study.
The added value of FS assessment is that it gathers information on the patient's psychosocial status. In the study case, the patient declared that she could not cope with her psychological problems. In addition, symptoms indicating depression were confirmed using the Centre for Epidemiological Study of Depression (CES-D) scale, a tool that possesses good psychometric properties. Can psychosocial burden affect the results of cardiac surgery? The coexistence of depression and cardiovascular diseases is common. When qualifying a patient for cardiac surgery, most of the data gathered concern the occurrence of post-operative psychiatric disorders. Depression symptoms are found in 25-35\% of patients undergoing cardiac surgery. Interestingly, epidemiological data indicate the presence of depression in $36 \%$ of patients awaiting coronary-artery bypass-graft surgery [21]. Depression or the risk of depression before cardiac surgery are associated with higher risk of post-operative depression, which has itself been shown to be an independent predictor of subsequent cardiac events, the risk of rehospitalisation, and, in the case of severe depressive disorders, the risk of death. Routine screening for depression symptoms is indicated as a standard part of the process of qualifying patients for cardiac surgery. Patients whose pre-operative symptoms indicate depression may be perceived as being more physically and emotionally vulnerable to the stresses of the surgical intervention than non-depressed cohorts [22]. Studies have confirmed the importance of social-support factors in the occurrence of depression in the elderly. Likewise, social support is a positive factor for post-operative health recovery. The six-month mortality rate is lower in elderly patients who have social or religious support than in patients without similar support [1].

Frailty in the social domain significantly correlates with a reduced level of self-control and self-care in heart failure patients. With regard to the question posed earlier, it should be recognised that the psychosocial burden may affect the results of cardiac surgery, in particular, 
the patient's adherence to post-operative recommendations. In addition, their psychosocial status may further limit older people's ability to make decisions [5]. In the study case, the patient was assessed for cognitive function using the MMSE questionnaire. This assessment is important because elderly people with dementia, who undergo cardio-surgical procedures, are four times more likely to experience post-operative complications than are patients without dementia [1]. Furthermore, in both clinical practice and scientific research, assessing mental functioning helps to identify patients who do not have the ability to give informed consent [19]. It should be emphasised that the incidence of dementia in the elderly increases the inability of patients in this group to make decisions. The following factors can impede the ability of patients to provide true consent: feeling threatened by illness, fear, lack of education, inability to properly understand the language, belief that there is no other treatment option, and the presence of mental disorders [1].

According to ethical principles, the clinician is obligated to protect patients who lack the ability to make decisions against inappropriate decisions regarding medical care. In many cases, patients with mild cognitive impairment have the ability to make decisions. Such patients can understand the indications for the planned procedure, the risks, and the benefits. Specialists who can help determine the patient's ability to make decisions include psychiatrists, geriatricians, clergy, social workers, and other members of the therapeutic team [5]. Informed consent, which is the main manifestation of respect for the patient's autonomy, includes the following components: (1) pre-conditions (capacity, voluntariness), which include the need to assess the patient's ability to understand information and make a voluntary and autonomic decision; (2) information (disclosure, recommendations), which includes the need to present to the patient all the information necessary to make decisions, including the nature of the disease, treatment options, risks, benefits, potential consequences of therapy, prognosis without treatment, and recommendation of a specific treatment plan; and (3) consent (decision, authorisation), which includes making a decision on the basis of the information provided, which is adequate to the clinical situation, consistent with the patient's preferences and values, and confirmed by written authorisation to perform the procedure [9].

Depressive symptoms, a patient's level of social support, and his or her cognitive functioning all are determinants of FS, which, as discussed earlier, may influence the effectiveness of cardiac surgery. It is a violation of the informational component of informed consent if the patient is not presented with the risk embodied in the assessment of FS during the qualification process. Violation of any of the three components of informed con- sent may invalidate the consent obtained. Similarly, the patient's signature would constitute a falsely understood consent. It should be emphasised that giving consent is a process in which both the patient and the clinician play important roles [9]. It is the moment in which to consider the possible consequences of the decision [23]. When the planned procedure carries a high risk of complications or even death and a low chance of improving the patient's quality of life, it is particularly important that the decision-making process includes the opinions of all members of the therapeutic team, including the nurse as a patient advocate [10]. Medical interventions that do not improve the patient's quality of life in the biopsychosocial sense are often futile and contrary to basic human dignity. The quality-of-life question makes it even more important to include the patient and his or her family in the medical decision-making process, because the quality of life that the healthcare team members may perceive as low may be perceived as good or fully acceptable by the patient and his or her family [10].

The presence of FS can change the decision-making regarding treatment options. FS patients may benefit from the heart team-based approaches to care [2, 24]. Hill et al. [19] proposed a comprehensive model of risk assessment and a course for the decision-making process for FS patients. Scientific evidence confirms the negative effect of embrittlement on both the quality of life and the life expectancy of patients after cardiac surgery, supporting the implementation of an appropriate, unified model of qualification for daily clinical practice.

Prehabilitation, a new concept in multidisciplinary care for patients preparing for planned cardio-surgical procedures, is increasingly being recognised as a valuable part of the qualifying process for patients with FS [3]. Prehabilitation aims to optimally use the patient's time while awaiting the procedure by providing him or her with multidisciplinary consultations and using physical and mental training and nutritional support to optimise his or her condition. A randomised trial that included low-risk patients qualified for coronary-artery bypass-graft surgery found that 10 weeks of pre-operative physical training was associated with shorter stays in both the intensive care unit and the hospital after the procedure [24]. Education is vital to prehabilitation. It must be noted that the frailty of the patient in the study case might have been modifiable using prehabilitation. Numerous studies are currently underway on prehabilitation, which may prove to be particularly helpful to FS patients. Identification of FS may cause ethical anxiety, but it does not automatically disqualify a patient from undergoing cardiac surgery; however, FS does require the correct approach to the decision-making process, in which a nurse speaking in the patient's best interests as an advocate can play a significant role. 
In conclusion, an increasing number of scientific studies confirm the necessity of including FS in models of peri-operative risk prognosis and in the decision-making process used in daily clinical practice. A patient's poor psychosocial status significantly increases his or her peri-operative risks, including those of increased morbidity, mortality, institutionalisation, disability, and loss of independence. If the patient is not presented with a risk assessment that includes FS assessment during the qualification process, it can be concluded that this omission violates the information component of informed consent. As regards the healthcare team, a reliable assessment of peri-operative risk is vital to making ethical clinical decisions. One element important to preventing or mitigating ethical dilemmas during the decision-making process is effective communication among members of the multidisciplinary team. Because nurses are the team members who are tasked with the role of advocating for their patients, they must constantly be aware of their patients' biopsychosocial statuses and speak out in those patients' interests in order to preclude actions that may increase their vulnerability during cardiac surgery.

\section{Summary}

This paper presents the state of knowledge on the most important issues related to qualifying patients with FS for cardiac surgery. The issues presented are discussed in the context of the emergence of possible ethical dilemmas in daily clinical practice. Therefore, this paper has both practical and substantive indications for people involved in cardiac surgery. It also discusses nurses' active participation in the process of making decisions regarding cardiac surgery.

\section{Disclosure of interest}

The authors declare that they have no conflict of interest.

\section{Acknowledgements}

The authors received no specific funding for this work.

\section{References}

1. Hecker S, Sade R. Ethical Issues in Cardiothoracic Surgery for the Elderly. Cardiothoracic Surgery in the Elderly. 2011: 89-103, doi: 10.1007/978-1-4419-0892-6_8

2. Vetta F, Locorotondo G, Vetta G, et al. Prognostic impact of frailty in elderly cardiac surgery patients. Monaldi Arch Chest Dis. 2017; 87(2): 855, doi: 10.4081/monaldi.2017.855, indexed in Pubmed: 28967721.
3. Hubbard RE, Story DA. Patient frailty: the elephant in the operating room. Anaesthesia. 2014; 69 Suppl 1: 26-34, doi: 10.1111/anae.12490, indexed in Pubmed: 24303858

4. Allen KB. Frailty: it's hard to define, but you know it when you see it. J Thorac Cardiovasc Surg. 2014; 148(6): 3117-3118, doi: 10.1016/j. jtcvs.2014.10.013, indexed in Pubmed: 25433887.

5. Mueller PS, Hook CC, Fleming KC. Ethical issues in geriatrics: a guide for clinicians. Mayo Clin Proc. 2004; 79(4): 554-562, doi 10.4065/79.4.554, indexed in Pubmed: 15065621.

6. Ridderstolpe L, Collste $\mathrm{G}$, Rutberg H, et al. Priority setting in cardiac surgery: a survey of decision making and ethical issues. J Med Ethics. 2003; 29(6): 353-358, doi: 10.1136/jme.29.6.353, indexed in Pubmed: 14662815

7. Stone ML, Kern JA, Sade RM. Transcatheter aortic valve replacement clinical aspects and ethical considerations. Ann Thorac Surg. 2012; 94(6): 1791-1795, doi: 10.1016/j.athoracsur.2012.09.047, indexed in Pubmed: 23176901

8. Sepehri A, Beggs T, Hassan A, et al. The impact of frailty on outcomes after cardiac surgery: a systematic review. J Thorac Cardiovasc Surg. 2014; 148(6): 3110-3117, doi: 10.1016/j.jtcvs.2014.07.087, indexed in Pubmed: 25199821

9. Kavarana MN, Sade RM. Ethical issues in cardiac surgery. Future Cardiol. 2012; 8(3): 451-465, doi: 10.2217/fca.11.91, indexed in Pubmed: 22642634

10. Kirsch RE, Coronado J, Roeleveld PP, et al. The Burdens of Offering: Ethical and Practical Considerations. World J Pediatr Congenit Heart Surg. 2017; 8(6): 715-720, doi: 10.1177/2150135117733940, indexed in Pubmed: 29187107

11. Gagnier JJ, Kienle G, Altman DG, et al. The CARE guidelines: consensus-based clinical case reporting guideline development. BMJ Case Rep. 2013; 2: 38-43.

12. Fried LP, Tangen CM, Walston J, et al. Cardiovascular Health Study Collaborative Research Group. Frailty in older adults: evidence for a phenotype. J Gerontol A Biol Sci Med Sci. 2001; 56(3): M146-M156, doi: 10.1093/gerona/56.3.m146, indexed in Pubmed: 11253156.

13. Gobbens RJJ, van Assen MA, Luijkx KG, et al. The Tilburg Frailty Indicator: psychometric properties. J Am Med Dir Assoc. 2010; 11(5): 344-355, doi: 10.1016/j.jamda.2009.11.003, indexed in Pubmed: 20511102 .

14. Uchmanowicz I, Jankowska-Polańska B, Łoboz-Rudnicka M, et al Cross-cultural adaptation and reliability testing of the Tilburg Frailty Indicator for optimizing care of Polish patients with frailty syndrome. Clin Interv Aging. 2014; 9: 997-1001, doi: 10.2147/CIA.S64853, indexed in Pubmed: 25028543.

15. Tomaschewski-Barlem JG, Lunardi VL, Barlem ELD, et al. Patient advocacy in nursing: barriers, facilitators and potential implications. Texto Contexto Enferm. 2017; 26: 117.

16. Davoodvand S, Abbaszadeh A, Ahmadi F. Patient advocacy from the clinical nurses' viewpoint: a qualitative study. J Med Ethics Hist Med. 2016; 9: 5, indexed in Pubmed: 27471588

17. Yanagawa B, Latter DA, Fedak PWM et al. The Cost of Frailty in Cardiac Surgery. Can J Cardiol. 2017; 33(8): 959-960, doi: 10.1016/j. cjca.2017.05.015, indexed in Pubmed: 28666616.

18. Afilalo J, Mottillo S, Eisenberg MJ, et al. Addition of frailty and disability to cardiac surgery risk scores identifies elderly patients at high risk of mortality or major morbidity. Circ Cardiovasc Qual Outcomes. 2012 5(2): 222-228, doi: 10.1161/CIRCOUTCOMES.111.963157, indexed in Pubmed: 22396586.

19. Hill A. Alkeridy. Preoperative assessment of the frail older adult. $\mathrm{Br}$ Columbia Med J. 2017; 59: 112-116.

20. Morley JE, Vellas B, van Kan GA, et al. Frailty consensus: a call to action. J Am Med Dir Assoc. 2013; 14(6): 392-397, doi: 10.1016/j. jamda.2013.03.022, indexed in Pubmed: 23764209.

21. Patron E, Messerotti Benvenuti S, Palomba D. Preoperative and perioperative predictors of reactive and persistent depression after cardiac surgery: a three-month follow-up study. Psychosomatics. 2014; 55(3): 261-271, doi: 10.1016/j.psym.2013.12.011, indexed in Pubmed: 24673940.

22. Horne D, Kehler S, Kaoukis G, et al. Depression before and after cardiac surgery: do all patients respond the same? J Thorac Cardiovasc Surg. 2013; 145(5): 1400-1406, doi: 10.1016/j.jtcvs.2012.11.011, indexed in Pubmed: 23260432

23. Nurok M, Makkar R, Gewertz B. The Ethics of Interventional Procedures for Patients Too III for Surgery. JAMA. 2017; 317(4): 359-360, doi: 10.1001/jama.2016.20288, indexed in Pubmed: 28118464.

24. Graham A, Brown CH. Frailty, Aging, and Cardiovascular Surgery. Anesth Analg. 2017; 124(4): 1053-1060, doi: 10.1213/ANE.0000000000001560, indexed in Pubmed: 27622718 